\title{
Evaluation of adhesive bonding of ceramic tiles using active thermography
}

by N. Simões*, I. Simões* ${ }^{*}$ A. Tadeu* and C. Serra**

\author{
${ }^{*}$ CICC, Department of Civil Engineering, Faculty of Sciences and Technology, University of Coimbra, Rua Luís Reis Santos \\ - Pólo II da Universidade, 3030-788 Coimbra, Portugal, \{nasimoes, mivsimoes, tadeu\}@itecons.uc.pt \\ **ITeCons, Rua Pedro Hispano, Pólo II da Universidade, 3030-289 Coimbra, Portugal, cserra@itecons.uc.pt
}

\section{Abstract}

Adhesion failure and/or detachment of ceramic tiling are common building defects. Bonding rupture of this type of coating presents a serious risk to people's safety, in particular if it occurs on external façades. It may be avoided by earlier non-destructive inspections.

This paper presents the results of an active thermography investigation in which adhesive bonding defects of ceramic coatings were examined. A set of non-destructive results was gathered based on a set of defects induced in the adhesive layer and an inappropriate adhesive technique or forced detachment between the ceramic tile and the bed layer.

\section{Introduction}

The reliable performance of some structural and construction materials, composite structural systems and construction elements is affected by internal defects. The early detection and characterization of cracks, voids and other classes of defects is essential to prevent construction pathologies, and material, or even structural failure. Efficient and consistent non-destructive evaluation techniques (NDTs) can be explored when the state or intended function of a system needs to be determined without the use of invasive approaches. In building construction, the application of NDTs has been found useful in the detection of internal voids, delaminations and cracks in concrete structures such as bridge decks and highway pavements as well as in walls and floors of buildings. Many non-destructive testing methods are available. Ideally a non-destructive technique should reliably detect the smallest flaw that is of concern, and be able to inspect large areas as well as smaller localized areas. Furthermore, it must be efficient in terms of labour and equipment, unobtrusive to the surrounding environment and convenient to users of the structure.

Heat diffusion is found to be sensitive to some defects in building elements, therefore the Infrared Thermography (IRT) technique has proved to be a powerful applicable non-destructive inspection for detecting heat losses, missing or damaged insulation, thermal bridges, air leakages and excess moisture ([1]-[5]) and for testing the integrity of composites or structures ([6]-[10]). IRT can be divided into the passive and active approaches. In the active IRT the object under study is subjected to an external controlled heat diffusion process, while in the passive approach materials and structures are normally exposed to a natural temperature gradient. In the first approach it should be possible to get repeatable results. As described by Meola [11], the detection of defects depends on three main factors: geometrical, thermal and measured factors. The first factor includes the area and thickness of the defect, while the second factor is relative to the thermal characteristics (thermal conductivity, diffusivity and effusivity) of both the defect and the hosting material. The last factor includes the characteristics of the used apparatus and test parameters, in particular the sensitivity of the IR camera.

Besides the two approaches to induce thermal response, there are two ways to trigger heat transfer phenomena from the object under study. The first is based on a transmission process where the stimulus is induced behind the intended viewing area and the response is dependent on the body thermal characteristics, while in the second the stimulus is applied to the front and viewed surface (reflection process).

Depending on the external stimulus, different approaches of active thermography have been developed, such as pulse thermography, lock-in thermography, step heating and vibrothermography [12]. The success of the analysis is dependent of the right choice of stimulus, how thermal stimulation is performed (transmission or reflection) and the sensitivity of the infrared imaging system.

Wall cladding systems used on exterior building envelopes may consist of heavy materials such as stone panels or ceramic tiles. Ceramic tiles are still largely used in Portugal as a decorative and functional cladding of exterior façades (see Fig.1). Most of the time, the cladding material is applied directly on the support using an adhesive. However, adhesion failure and/or detachment of ceramic tiling are common building defects (see Fig. 2). This represents a risk to people's safety, especially in the case of larger ceramic tiles, thus special attention must be given to the application phase ([13]-[15]). The risk of detachment of these cladding materials should be properly assessed, in particular in high buildings. These buildings require early and periodic inspections in order to evaluate existing conditions and uncover potential deficiencies so as to prevent catastrophic failure. To avoid invasive inspections, non-destructive 
and non-contact techniques such as IRT may be used [10]. However, we may notice that the detection of a defect depends on several factors such as the defect geometry (area and thickness), depth and orientation in relation to the surface under inspection, and thermal properties of the defect and hosting medium.

In this work the feasibility of lock-in active IRT is explored to determine the capabilities and limitations of using IRT to assess the risk of bonding failure of ceramic cladding. Therefore an experimental campaign is needed to evaluate if IRT can detect adhesion defects or detachment of ceramic tiling. In this work, the specimens with induced defects are heated using halogen lamps. The object's surface temperature as a function of time is measured using an IRT camera - FLIR A615. The stored data is then analysed using software developed by Automation Technology. As a result, the collected data can be used to evaluate factors such as the adhesive technique (total spreading and glue fixation points), different sizes of induced defects and coating with different emissivities.

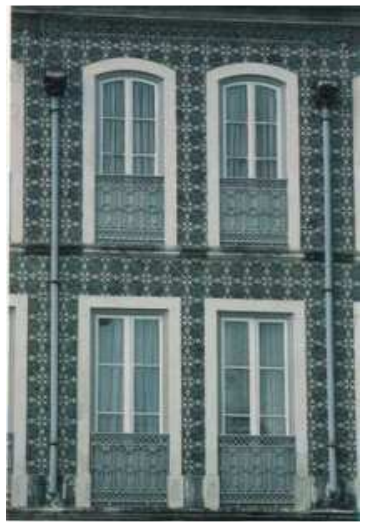

Fig. 1. Portuguese ceramic cladding façade.

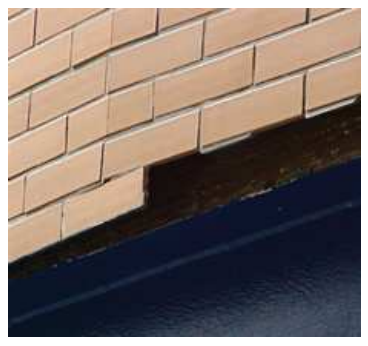

Fig. 2. Detachment of ceramic tiling.

\section{Experimental apparatus and test procedure}

\subsection{Description of apparatus}

The tests were carried out using a modular solution that supports the lock-in thermography technique, developed by Automation Technology. The system is composed of two halogen lamps, used as a heat source placed in front of the object. When switched on, heat sources stimulate the flow of thermal energy through the object and the surface temperatures may be obtained using an infrared camera and thermal responses recorded as a function of time. Then, the data is processed and may be displayed as an image or mathematically worked to obtain temperature patterns that differentiate the internal defects. The modular solution is composed of a computer connected to a box (interface hardware package) that controls the IR camera and the excitation source, as shown in Fig. 3. It allows an exact synchronization between the camera and the excitation source. The measurements are automatically stored and further processed by the software IRControl version 4.5.9 developed by Automation Technology. The software offers some imaging algorithms for the evaluation of the resulting images aiding the detection of defects. The evaluation methods support measurements in reflection as well as in transmission arrangements. As the measured thermal response and measurement parameters are stored, it is possible to apply different evaluation methods later. The available Lock-in evaluation methods are "single frequency Fourier transformation", "harmonic approximation" and "harmonic approximation with analytic approach".

All of the thermography measurements mentioned below were taken with an infrared video camera, model A615 from FLIR Systems. The IR camera was equipped with focal length of $24.5 \mathrm{~mm}$ and a detector with $640 \times 480$ pixels resolution. This camera is able to detect a temperature difference of $0.05^{\circ} \mathrm{C}$ at $30^{\circ} \mathrm{C}$ and has an accuracy of $\pm 2^{\circ} \mathrm{C}$ or $2 \%$ of readings. 


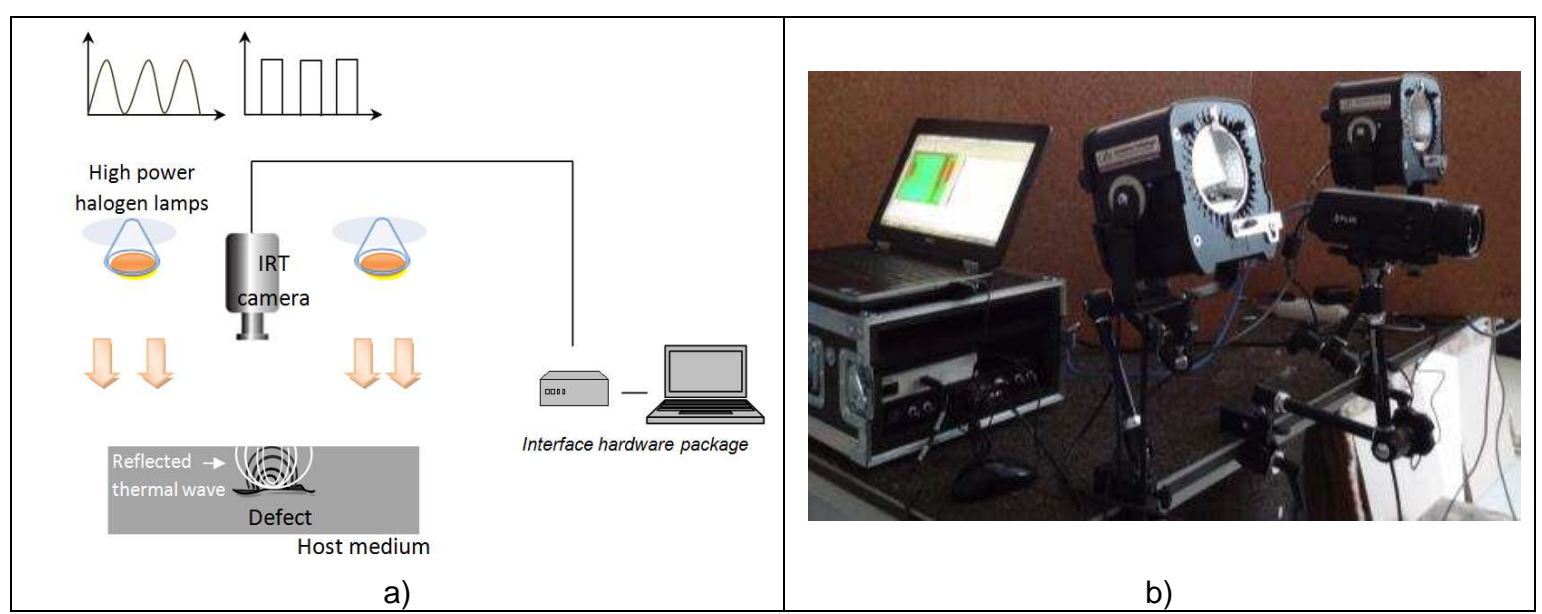

Fig. 3. Infrared imaging system used in the non-destructive evaluation with active thermography: a) Lockin system scheme with halogen excitation sources; b) Lock-in apparatus view (2 halogen lamps of $2.5 \mathrm{~kW}$; interface hardware package; infrared camera - model A615 from FLIR Systems).

\subsection{Description of specimens}

Four models of direct adhered ceramic tiles were prepared, for simulating the presence of defects and also to test inappropriate adhesion or induced partial detachment of the adhesive layer.

Schematic drawings of the four models are shown in Fig. 4 and 5, complemented with digital photos of the tile bed (adhesive mortar layer). In the case of Models 1 and 2 the dimensions and positions of the induced defects are presented, while in Model 3 the triggered detachment is shown. In Model 4 an inappropriate but common application is illustrated, with four glue points instead of a total spreading.

All models were prepared using three materials: dense cement wood board as substrate, cimentitious adhesive mortar, and ceramic tiles $12 \mathrm{~mm}$ thick. In the case of Model 2 the two smallest defects were left inside the tile bed to ensure the size of the defect doesn't change. In Model 3 half of the area of the adhesive mortar was covered by a polyethylene film, avoiding the adhesion between ceramic tile and the mortar. During the specimen's preparation, an application gauge with $5 \mathrm{~mm}$ was used to achieve the same tile bed thickness in all specimens (see Fig. 6a). Finally, the specimens were cured at a controlled temperature $\left(23 \pm 2^{\circ} \mathrm{C}\right)$ and relative humidity $(50 \pm 5 \%)$ for three weeks before experimental tests.

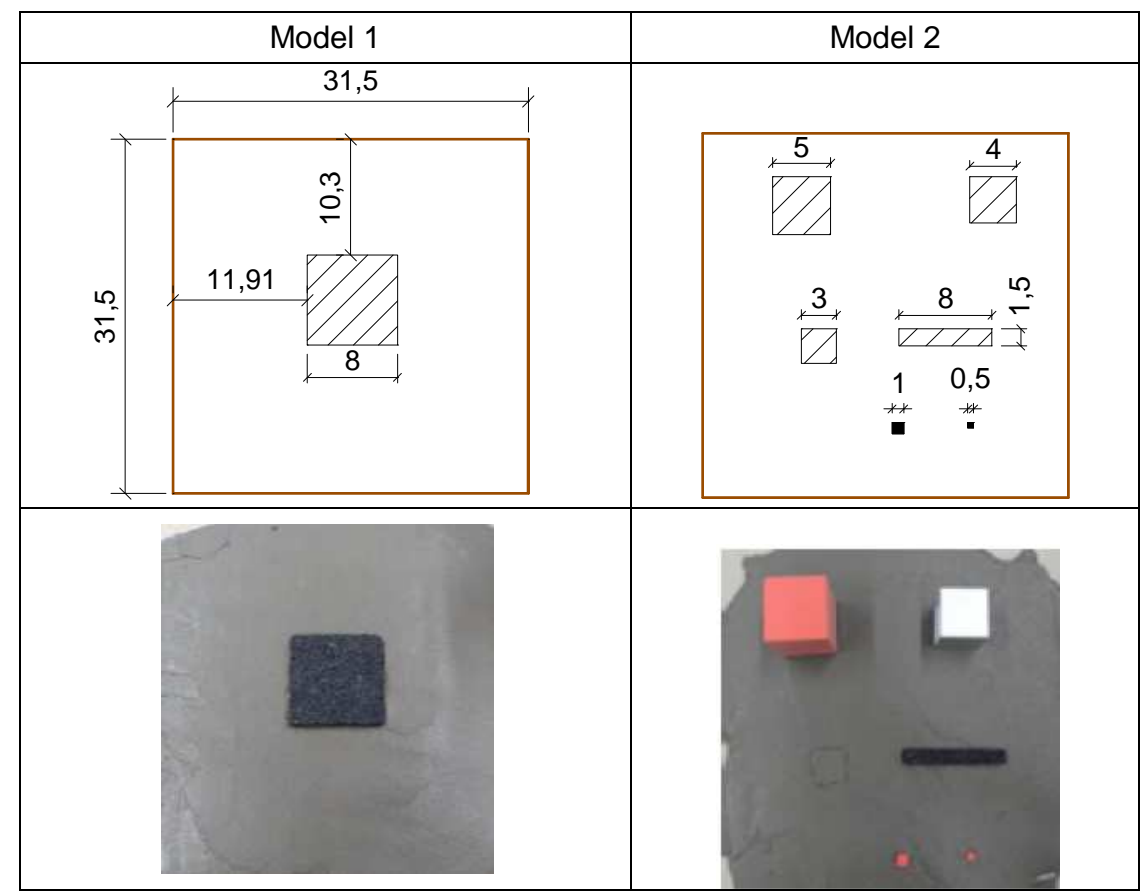

Fig. 4. Schematic drawing and digital photos of the studied ceramic specimens with induced defects. Geometry (dimensions in $\mathrm{cm}$ ) and position of the defects of Models 1 and 2. 


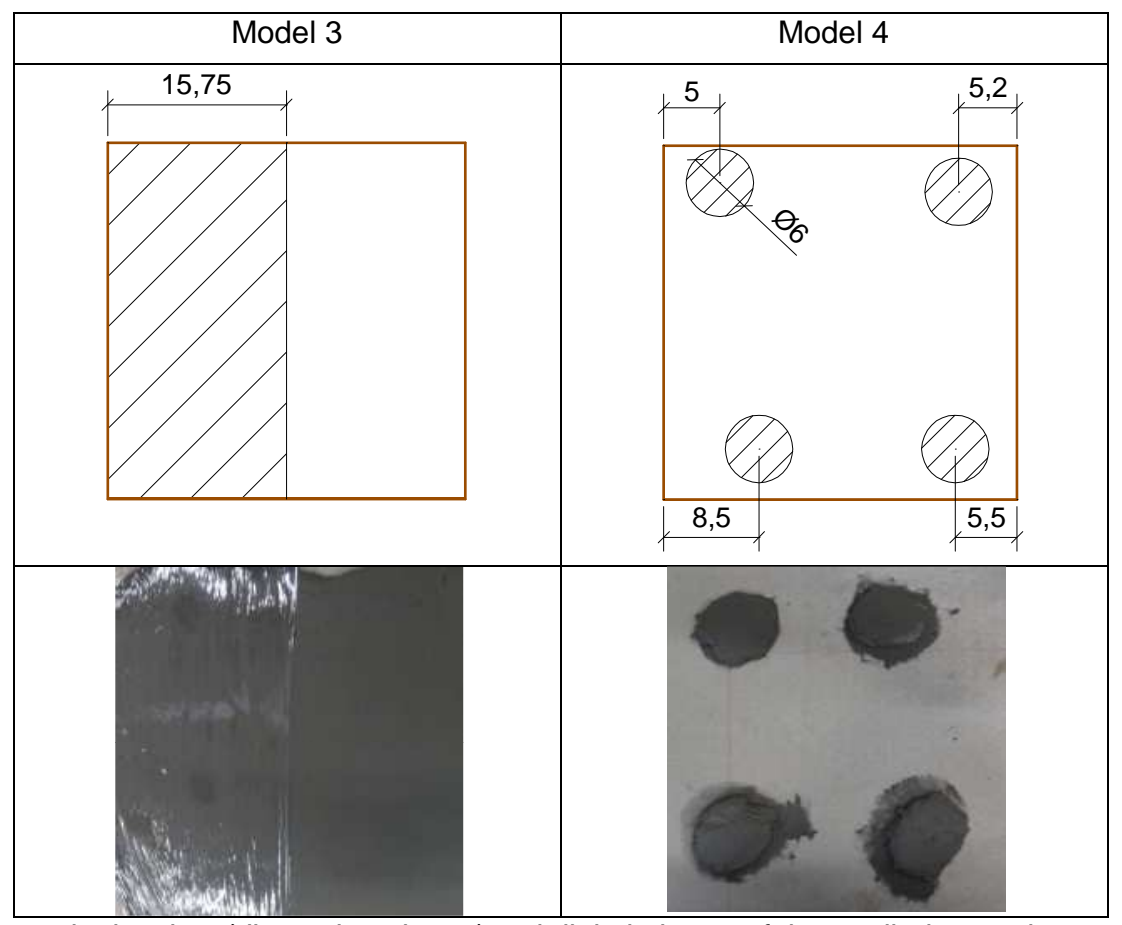

Fig. 5. Schematic drawing (dimensions in $\mathrm{cm}$ ) and digital photos of the studied ceramic specimens with induced partial detachment (Model 3) or inappropriate adhesion (Model 4).

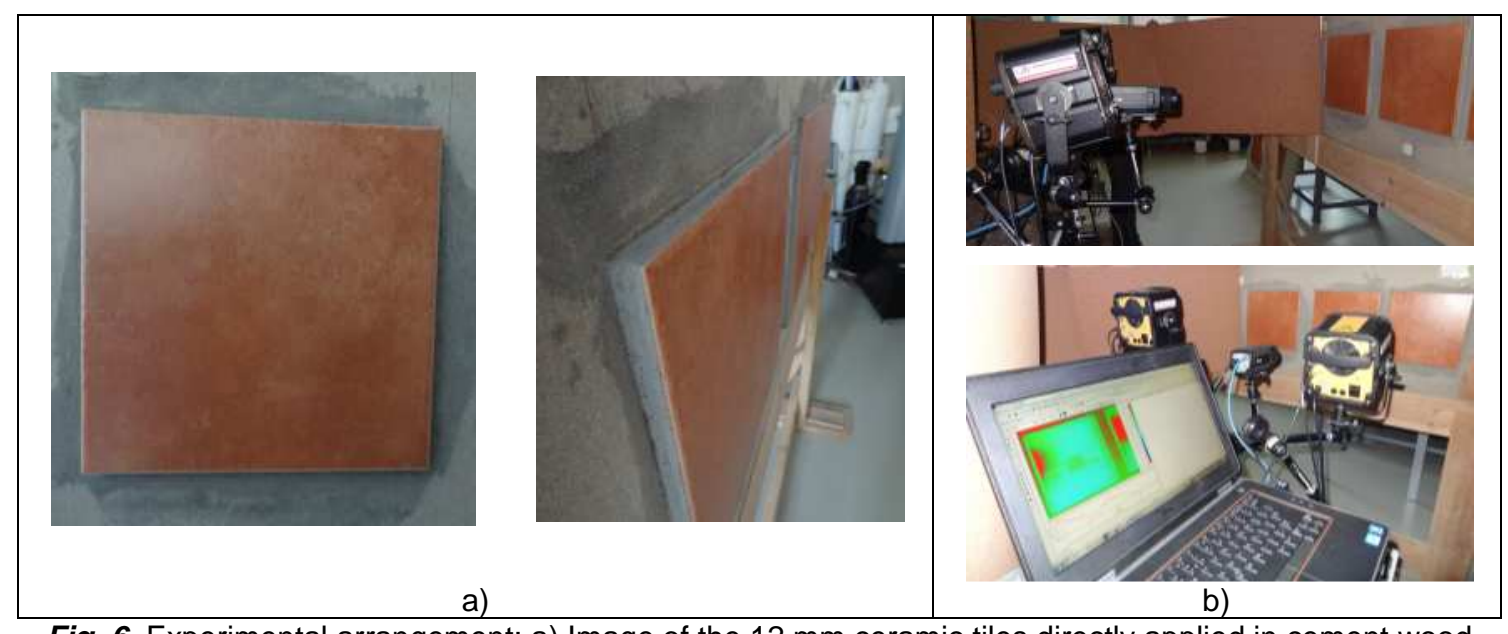

Fig. 6. Experimental arrangement: a) Image of the $12 \mathrm{~mm}$ ceramic tiles directly applied in cement wood board; b) Relative position of full components of the experimental scheme.

\subsection{Test procedure}

The measurements were carried out in a laboratory with controlled temperature and relative humidity. Test were performed using the video camera A615 from FLIR Systems, that was placed in front of the vertical substrate boards with the ceramic tiles applied on it, as shown in Fig. 6b. All the tests were performed in reflection mode with both halogen lamps and IR video camera positioned on the same side. The distance between the specimens and the IR camera/heat sources varied between $0.5 \mathrm{~m}$ and $0.7 \mathrm{~m}$.

The heat excitation was achieved with the two halogen lamps imposing a maximum power of $2.0 \mathrm{~kW}$ each $(80 \%$ of the available power). The source power (halogen lamps) kept constant for a total duration of 30 seconds, followed by a cooling phase of 60 seconds. The number of heating periods was no more than three. The data was recorded in video mode using image capture with a frequency of $12.5 \mathrm{~Hz}$.

\subsection{Thermal response analysis}

The experimental campaign carried out in this work consisted of a set of preliminary tests to explore and to evaluate if lock-in thermography is adequate for this kind of application. During tests, a video was recorded from which a set of thermal images was collected during the heating process and also during the cooling phase. 
Fig. 7 presents snapshots of Model 1 at three times: before heating; immediately after the first heating phase; and, during the cooling phase. Analyzing the first image, it appears that the defects are not detectable. After the application of the lock-in excitation it was possible to identify the defect represented in Fig. 4, identified in Fig. 7 by the number (1). The appearance of this defect is more evident in the cooling phase. Other changes in the temperature pattern are visible on the right side of the induced defect, marked with the number (2). To better understand this thermal response, the ceramic tile was removed after tests and is shown in Fig. 9a). A reduction of mortar thickness is visible, explaining why a change in the temperature pattern was detected.

As can be seen in Fig.8, related to Model 2, it was possible to detect some of the defects. With the IR camera placed $70 \mathrm{~cm}$ away from the object (allowing the view of the entire ceramic tile) it was possible to identify 4 out of the 6 induced defects (number (1) to (4)). The smallest defects, with $1.0 \mathrm{~mm}$ and $0.5 \mathrm{~mm}$ size, which were filled with a plastic material, were not detected. In a second test the IR camera was placed at only $50 \mathrm{~cm}$ away from the object to perform a more detailed analysis of the smaller defects. In this approach, it was possible to better identify defects (3) and (4), having detected a change in the pattern of temperatures between them. It follows that the smaller defects were not visible in the thermogram, although it identifies a very slight temperature change in the defect area $1 \mathrm{~cm}$ from the side (shown with number (5)). To better understand the thermograms the ceramic tile was removed and it was found that the defect with a size of $8.0 \mathrm{~cm} \times 1.5 \mathrm{~cm}$ had been divided into two during the bonding process (see Fig. 9b). This occurrence justifies the temperature change between points (3) and (4), cited above. It was also possible to detect the existence of areas with a lower mortar thickness on the right side, which led to higher temperatures, identified in Fig. 8 with the number (6).

Additionally a paper sticker with high emissivity was applied to the specimen of Model 2 in order to evaluate its influence on defect detection. In this case, no significant improvements were achieved with this surface change.

The results obtained for Models 3 and 4 are illustrated in Figure 10. Figure 10a) presents the Model 3 thermogram recorded immediately after the heating phase. The IR image does not give evidence of any perturbation of surface temperature distribution related to the induced defect. This conclusion can be confirmed by the temperature profile along the represented line. Figure 10b) shows the thermal response of Model 4 after the heating process where the four glue points are easily identified.

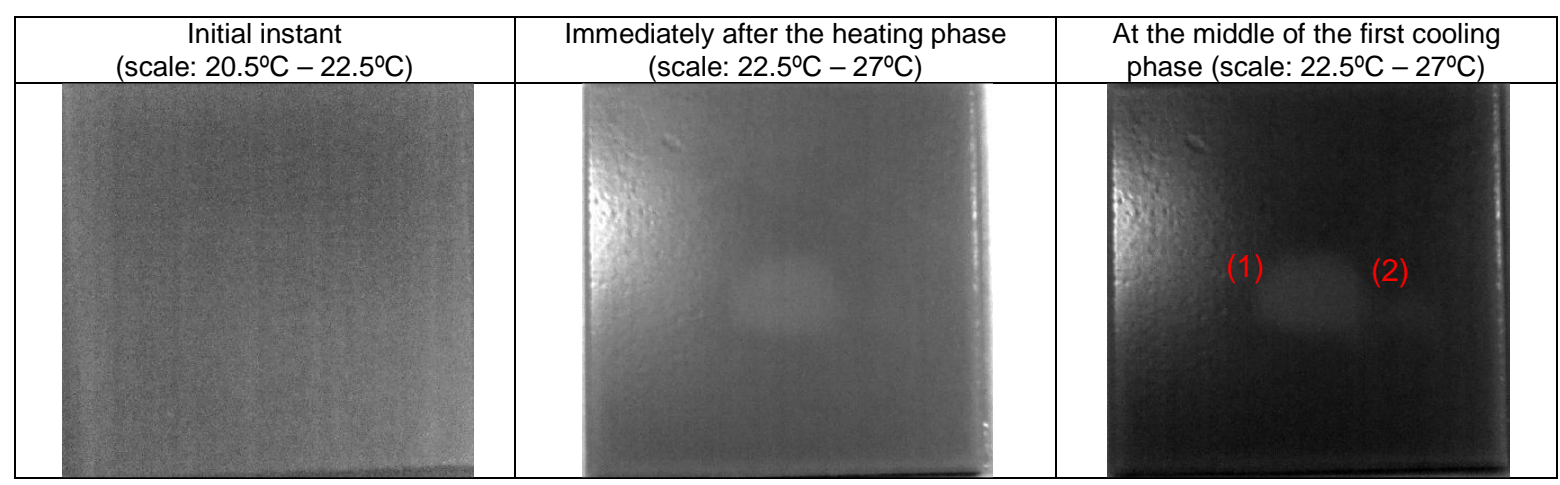

Fig. 7. Thermal images obtained during the experimental measurements of Model $1 \mathrm{IR}$ camera placed 70 $\mathrm{cm}$ away from the object surface.

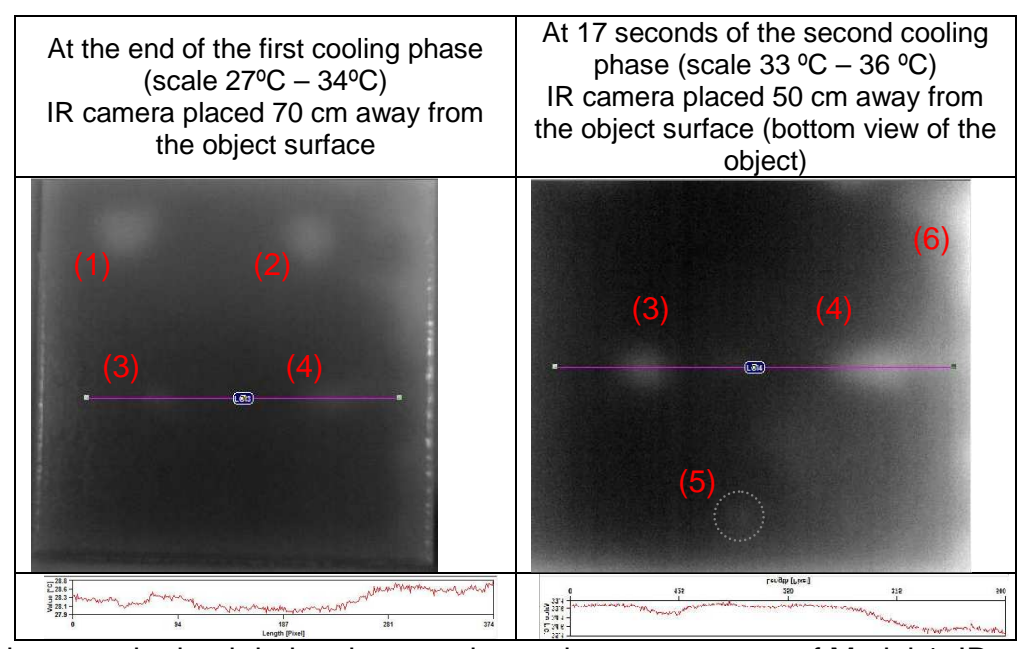

Fig. 8. Thermal images obtained during the experimental measurements of Model 1. IR camera placed 70 $\mathrm{cm}$ and $50 \mathrm{~cm}$ away from the object surface. 


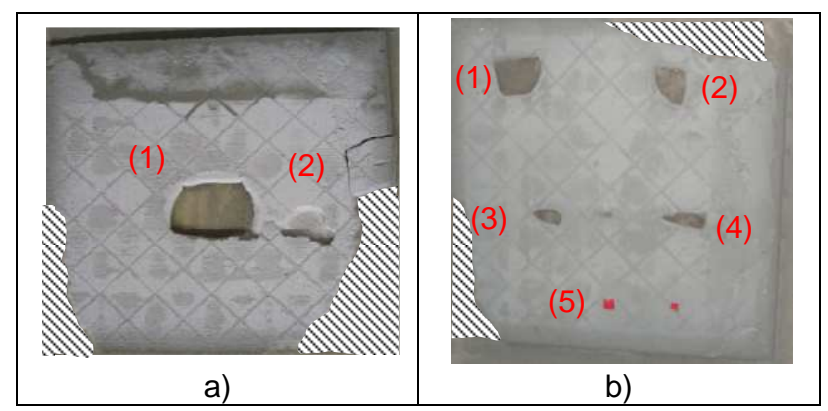

Lost material during the removal of the ceramic tile

Fig. 9. Tile bed after removing the ceramic tile: a) Induced defects of Model 1; b) Induced defects of Model 2.

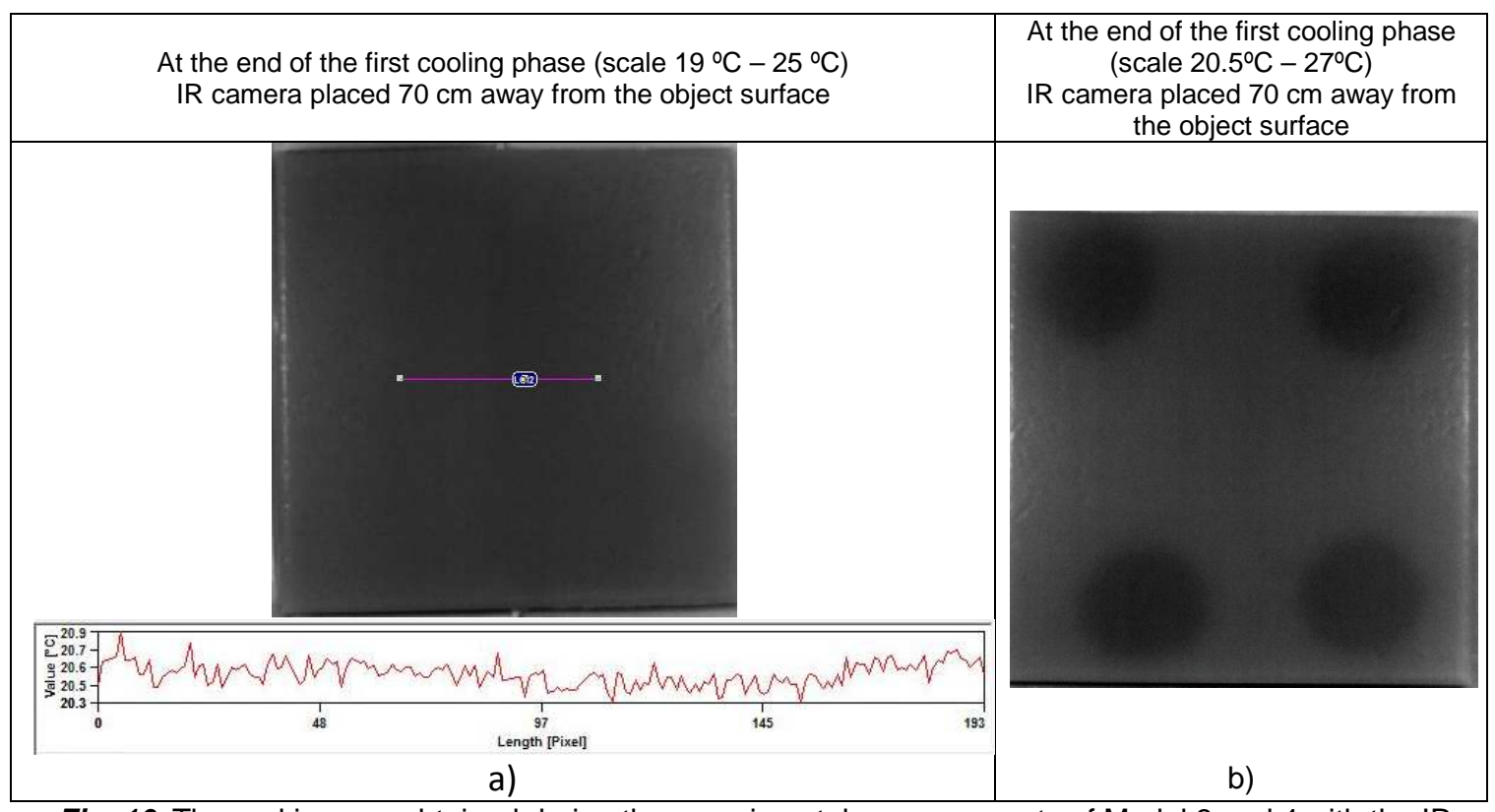

Fig. 10. Thermal images obtained during the experimental measurements of Model 3 and 4 with the IR camera placed $70 \mathrm{~cm}$ away from the object surface.

\section{Final remarks}

The knowledge of the fundamental parameters affecting heat transfer and infrared testing in ceramic tiles is essential to develop the foundation for this kind of thermographic inspection of building elements. This paper discusses the feasibility of using IR thermography to identify problems of the adhesive layer of ceramic coatings. Two models were used to analyse specific induced defects with different sizes, while the other two created inappropriate adhesion or induced partial detachment. Tests were performed in a controlled environment laboratory, using a lock-in system with halogen excitation sources and a video camera from FLIR. Images were recorded and analysed using a software package from Automation Technology.

The results obtained using the active infrared thermography technique could identify the defined defects and other unforeseen inclusions or clogging. Defects with a dimension larger than $1.5 \mathrm{~cm}$ were found to be easily detectable. The induced detachment was not detected, while the points of fixation were also easy to identify.

\section{ACKNOWLEDGMENTS}

The research work presented herein was supported by FEDER funds through the Operational Programme for Competitiveness Factors - COMPETE and by national funds through the FCT - Portuguese Foundation for Science and Technology), under research project PTDC/ECM/114189/2009.

The presented work is also framed under the Energy for Sustainability Initiative of the University of Coimbra, through the MIT PORTUGAL, supported by the FCT. 


\section{REFERENCES}

[1] Grinzato, E, V Vavilov, and T Kauppinen. Quantitative infrared thermography in buildings. Image Processing 29. 1998.

[2] Balaras, C A, and A A Argiriou. Infrared thermography for building diagnostics. Energy and Buildings 2002. 34: 171-183.

[3] Vavilov VP, Kauppinen T, Grinzato E. Thermal characterization of defects in buildings envelopes using long square pulse and slow thermal wave techniques, Res. Nondestr. Eval. 1997; 9: 181200.

[4] Jokisalo J, Kurnitski J, Korpi M, Kalamees T, Vinha J. Building leakage, infiltration, and energy performance analyses for Finnish detached houses, Building and Environment 2009; 44: 377387.

[5] Poblete A, Acebes A, Pascual M. Thermographic measurement of the effect of humidity in mortar porosity, Infrared Physics \& Technology 2007; 49: 224-227.

[6] Sham FC, Chen N, Long L. Surface crack detection by flash thermography on concrete surface. Insight 2008; 50(5):240-243.

[7] Maierhofer Ch, Arndt R, Rollig M. Influence of concrete properties on the detection of voids with impulse-thermography, Infrared Physics \& Technology 2007; 49: 213-217.

[8] Bjegovic D, Mikulic D, Sekulic D. Non-destructive methods for monitoring reinforcing steel in concrete, Proc. Struct. Faults and Repair 2001, London, CDROM 2001.

[9] Chenga C, Chenga T, Chiang C. Defect detection of concrete structures using both infrared thermography and elastic waves, Automation in Construction 2008; 18( 1): 87-92.

[10] Cerdeira F., Vázquez M.E., Collazo J., Granada E., Applicability of infrared thermography to the study of the behaviour of stone panels as building envelopes, Energy and Buildings, vol 43(8), pp 1845-1851, 2011.

[11] Carosena Meola, Infrared thermography of masonry structures, Infrared Physics \&amp; Technology, 49(3): 228-233, 2007.

[12] Maldague X., Introduction to NDT by Active Infrared Thermography, Materials Evaluation, 6(9): $1060-1073,2002$.

[13] Chew MYL, Factors affecting ceramic tile adhesion for external cladding, Construction and Building Materials, 13(5): 293-296, 1999.

[14] Mahaboonpachai T, Matsumoto T, Inaba $\mathrm{Y}$, Investigation of interfacial fracture toughness between concrete and adhesive mortar in an external wall tile structure, International Journal of Adhesion and Adhesives, 30(1): 1-9, 2010.

[15] Winnefeld F, Kaufmann J, Hack E, Harzer S, Wetzel A, Zurbriggen R, Moisture induced length changes of tile adhesive mortars and their impact on adhesion strength, Construction and Building Materials, 30:426-438, 2012. 